International Journal of Current Advanced Research

ISSN: O: 2319-6475, ISSN: P: 2319 - 6505, Impact Factor: SJIF: 5.995

Available Online at www.journalijcar.org

Volume 6; Issue 4; April 2017; Page No. 3263-3266

DOI: http://dx.doi.org/10.24327/ijcar.2017.3266.0241

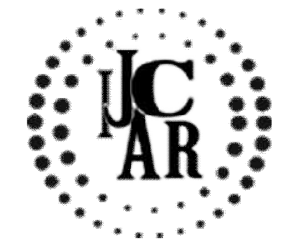

Review Article

\title{
THE FUTURE OF ANTIBIOTICS?- ICHIP AND TEIXOBACTIN-A REVIEW
}

\author{
Sandra Sagar ${ }^{*}{ }^{*}$, Ezhilarasan. ${ }^{2}$ and Sagar Moses $^{3}$
}

Saveetha Dental College and Hospitals, Chennai

\begin{tabular}{l}
\hline A R T I C L E I N F O \\
\hline Article History: \\
Received $20^{\text {th }}$ January, 2017 \\
Received in revised form $19^{\text {th }}$ February, 2017 \\
Accepted $22^{\text {nd }}$ March, 2017 \\
Published online $28^{\text {th }}$ April, 2017
\end{tabular}

Key words:

Teichoic Acid, Teixobactin,

Peptidoglycan

\begin{abstract}
A B S T R A C T
The development of antibiotic resistance is a major cause for concern in the health industry. The rate at which antibiotic resistance is developing is much faster than newer antibiotics are being introduced and if left unchecked may cause potential health crises in the coming years. Soil microorganisms makeup nearly all of the uncultured bacteria, and are a major source for potential new antibiotics. Synthetic antibiotics were unable to reproduce this as resistance developed faster. Teixobactin a new antibiotic isolated from an unknown Gramnegative bacterium that lives in the soil and which cannot be cultured in the lab using known techniques. Researchers fromLewis and Slave Epstein's lab developed a new and innovative approachcalled iChip that allowed the environmental bacterium to grow and for the antibiotic it produced to be isolated and subsequently identified. Teixobactin inhibits cell wall synthesis by binding to lipid II (precursor of peptidoglycan) and lipid III (precursor of cell wall teichoic acid). Binding of teixobactin to lipid precursors inhibits production of the peptidoglycan layer, leading to lysis of vulnerable bacteria.
\end{abstract}

Copyright $₫ 2017$ Sandra Sagar., Ezhilarasan.D and Sagar Moses. This is an open access article distributed under the Creative Commons Attribution License, which permits unrestricted use, distribution, and reproduction in any medium, provided the original work is properly cited.

\section{INTRODUCTION}

The development of antibiotic resistance is a major cause for concern in the health industry. The rate at which antibiotic resistance is developing is much faster than newer antibiotics are being introduced and if left unchecked may cause potential health crises in the coming years. Antibiotics were produced earlier by screening soil micro-organisms, but its limited resource and overkill led to its demise in 1960. Synthetic approaches to antibiotics production though successful initially later developed resistance and not cost effective. Our world contains a large pool of 'uncultivable 'microbial flora as only a fraction of this population grow in artificial media. One of the major challenges faced by microbiologists today therefore is to tap potential of this microbial diversity which is a promising source of new antibiotics. Several methods were therefore developed to cultivate 'uncultured 'organisms in their natural environment. Researchers from Lewis and Slave Epstein's lab developed a new and innovative approach called iChip that allowed the environmental bacterium to grow, and for the antibiotic it produced to be isolated and subsequently identified. Teixobactin is first of a new class of antibiotics discovered by ichip technology (Laura J. V. Piddock, 2015), [1] and a new antibiotic that kills pathogens without any detectable resistance (2015Ling et al..) [2]. This article reviews the importance of the new technique iChip and the discovery of the new antibiotic Teixobactin!.

*Corresponding author: Sandra Sagar

Saveetha Dental College and Hospitals, Chennai

\section{Development of iChip device}

For years the technique to grow bacteria in the lab remained the same and an overwhelming population of these bacteria refused to grow under those conditions. This is called the Great plate Anomaly whichis the observation that most of the microbes seen in the microscope cannot currently be grown under laboratory conditions, some may actually be nonviable, others are viable but nonculturable (VBNC) [3]

This means that the researchers were unable to study the chemicals these bacteria process which has antimicrobial properties which the bacteria may use to killother threatening microbes, and they could potentially be synthesized to make antibiotics. Many attempts to fix this growth problem failed.[4]

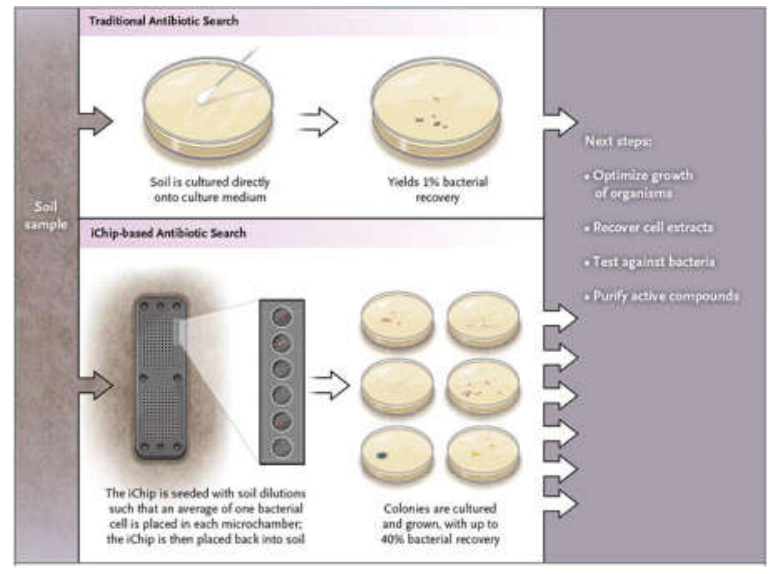


Slava Epstein the inventor of iChip and his colleague microbiologist Kim Lewis, had a new approach to this problem. They realised that the natural environment may contain molecules which are nutrient or growth factors which may provide messages that trigger bacteria to grow at the right time.Instead of knowing what these mystery components were they decided to bring the whole environment to the lab including these molecules.

The team, with Tammi Kaeberlein, developed a diffusion chamber, in which bacterial samples were sandwiched between a breathable material with pores that were big enough to let nutrients in and waste out, but small enough to contain the bacteria. They then placed it in bacteria's natural environment, (marine sediment)and set the chamber in the lab.[5]
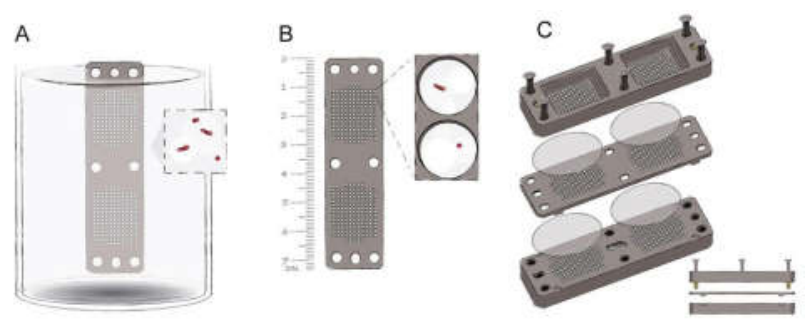

The device worked and allowed them to grow considerable amount of bacterial colonies compared to an agar plate. But the chamber didn't isolate a specific bacterial strain, but included many species at once. They then build a device that could isolate individual bacterial cells and allowed them to grow. With Dominica Nichols and Emil Trakhtenberg, an engineer at Argonne National Lab along with a team of technicians made a device which could cultivate dozens of samples simultaneously. This method not only identified the new species of microbes but also allowed them to multiply. This new device was known as iChip, which was a hard piece of plastic with 192 tiny wells. All the Scientist had to do was to dip the Ichip in the bacterial sample, mixed with agar which would trap cells in each well and secure diffusion membranes to lock the sample inside. This was then placed in a larger sample from its natural environment. Each cell then became a miniature diffusion chamber.Microbes grew from a wide range of sources like saliva, marsh, wastewater and mud. The proof-of-concept of the iChip was first published in 2010. Thousands of new bacteria have now been cultured by iChip, including the species that produced Teixobactin. $[2,5,6,7,8]$

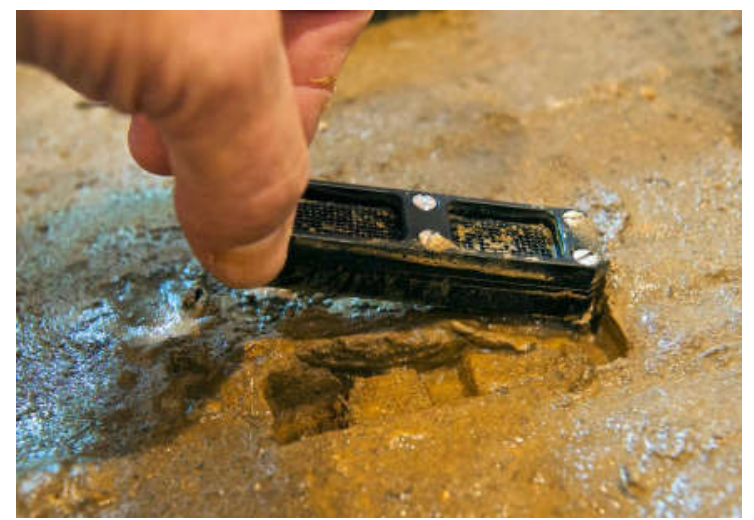

\section{Teixobactin}

Extracts from 10,000isolates obtained by growth in iChips were screened for antimicrobialactivity on plates overlaid with S. aureus.
An extract from a new speciesof b-proteobacteria provisionally named Eleftheria terrae showed goodactivity. The genome of E. terrae was sequenced. Based on 16S rDNA and in silico DNA/DNA hybridization, this organism belongs to a new genus related to Aquabacteria. This group of Gramnegativeorganisms is not known to produce antibiotics. [2]

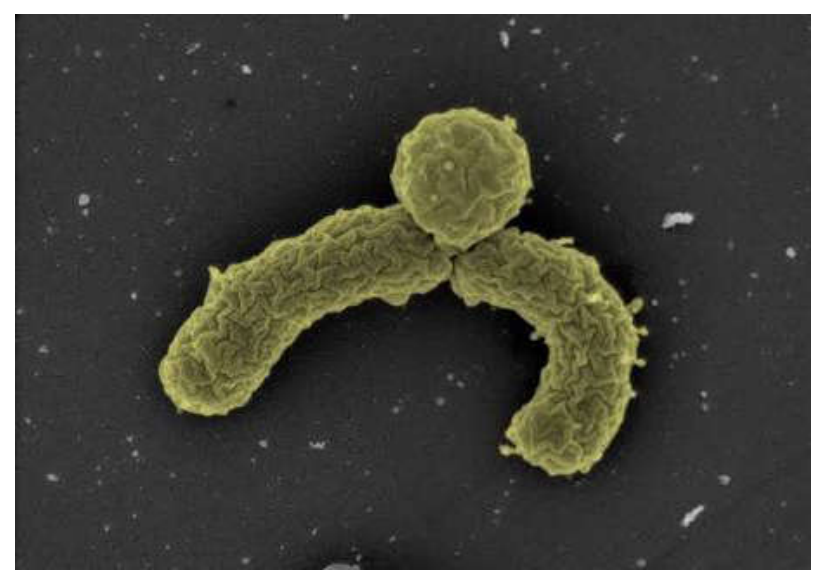

From the culture supernatant of E. terrae a partially purifiedactive fraction was obtained and shown to contain a compound, teixobactin. It is an unusual depsipeptide, containing enduracididine, methylphenylalanine and four Damino acids. The biosynthetic pathway of teixobactin was identified by genome sequencing of E. terrae and homology searches.[1] Image Eleftheria terrace, the Gram-negative bacterium that produces Teixobactin SEM picture courtesy William Fowle, M Northeastern University

\section{Resistance and Mechanism of action}

Teixobactin is the first member of a novel class of peptidoglycan synthesis inhibitors.[1] Teixobactin had excellent activity against Gram-positive pathogens,including drug-resistant strains. Potency against most species, including difficult-to-treat enterococciand M. tuberculosis was below 1nanogram ml. Teixobactin was exceptionally active against Clostridium difficile and Bacillus anthracis (minimalinhibitory concentration (MIC) of 5 and $20 \mathrm{ng} \mathrm{ml}$, respectively. 9,10

Teixobactin had excellent bactericidal activity against $\mathrm{S}$. aureus and was superior to vancomycin in killing late exponential phase populations, and retained bactericidal activity against intermediateresistance $\mathrm{S}$. aureus. The frequent clinical failure in patients with S. aureus MRSA treated withvancomycinhas been linked to the poor bactericidal activity of this compound. Teixobactin was ineffective against most Gram-negative bacteria, butshowed good activity against a strain of $E$. coliwith a defective outer membrane permeability barrier.

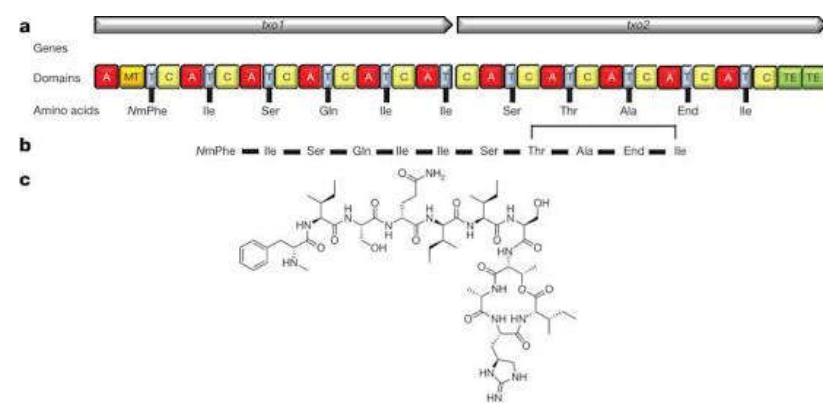




\section{The structure of teixobactin and the predicted bio synthetic} gene cluster

Mutants of S. aureus or M. tuberculosisresistant to teixobactin were not produced even when plating on media with a low dose of the compound. Serial passage of S. aureus in the presence of sub-MIC levels of teixobactin over a period of 27 days failed to produce resistant mutants as well. This usually means a non-specific mode of action, with accompanying toxicity. However, teixobactin had no toxicity against mammalianNIH/3T3 and HepG2 cells at $100 \mathrm{mgml}$, which was the highest dose tested. The compound showed no haemolytic activity and did not bind DNA. Inorder to determine specificity of action of teixobactin, its effect on the rateof label incorporation into the major biosynthetic pathways of S. aureus were examined. Teixobactin strongly inhibited synthesis of peptidoglycan, but had virtually no effect on label incorporation into DNA, RNA and protein. This suggested that teixobactin is a newpeptidoglycan synthesis inhibitor.

Resistance has not developed to this compound, suggesting that thetarget is not a protein. The essential lack of resistance development through mutations has been described for vancomycin which bindslipid II, the precursor of peptidogly can and teixobactin could be acting against the same target, lipid. Losee Ling from NovoBiotic Pharmaceuticals and Tanja Schneider at the University of Bonn showed that teixobactin works by with holding two molecules-Lipid II, which bacteria need to make the thick walls around their cells, and Lipid III, which stops their existing walls from breaking down. Teixobactin causes lysis of the cell wall which are not regenerated.

Treatment of whole cells of S. aureus with teixobactin (1-5 $\mathrm{xMIC}$ ) resulted in significant accumulation of the soluble cell wall precursor undecaprenyl-N-acetylmuramicacidpentapeptide (UDP-MurNAc-pentapeptide), similar to the vancomycin-treated control cells, showing that one of the membrane associated steps of peptidoglycan biosynthesis is blocked. Teixobactin inhibited peptidoglycan biosynthesis reactions in vitro in a dose-dependent manner with either lipid I, lipid II or undecaprenyl-pyrophosphate as a substrate. Teixobactin thus seemed safe, stable in blood, and capable of protecting mice from lethal doses of MRSA (drug resistant staphylococcus aureus).

The drug also sticks to parts of both Lipid II and Lipid III that are constant across different species of bacteria. It's likely that these parts can't be altered and can deflect teixobactin. So does E.terrae the microbe that makes the drug in the first place. Many of the resistance mutations that defuse antibiotics originate the microbes that produce those drugs in order to protect themselves. But since E.terrae is impervious to teixobactin, it doesn't need any such mutations and thus resistant. This compound is highly potent against a broad range of Gram-positive microbes, including methicillinresistant Staphylococcus aureus (MRSA). Teixobactin thus binds to two different targets - lipid II (peptidoglycan precursor) and lipid III (teichoic acid precursor). It binds to the undecaprenyl-PP-sugar region of these precursors, which is not known to be modified. As a result, teixobactin is the first example of a target-specific compound essentially free of resistance. Teixobactin shows excellent activity in several models of infection.
One of the major concerns of in the world of physicians today is antibiotic resistance in Gram-negative bacteria such as Escherichia coli and Klebsiella species. The Gram-negative bacterial cell envelope structure makes it difficultfor many antibiotics to gain entry into the bacterium and once inside many antibiotics are exported by multidrug efflux pumps. Ling et al. showed that teixobactin had no activity against E. coli, suggesting that E. coli is impermeable to this agent or it is effluxed (or both). Either way, teixobactin does notinhibit E. coli and so is unlikely to be effective against other Gramnegative bacteria.

\section{DISCUSSION}

World Health Organization in 2014 had warned that we were entering a 'post-antibiotic era'. United Kingdom had put antibiotic resistance on the government national risk register along with terrorists attacks and pandemics. This is so because no new class of antibiotics have been developed lately and only two class of antibiotics have been made since 1962.Globally deaths due to antibiotic resistance are estimated to be around 700,000 per year. Pharmaceutical companies have switched to more profitable ventures of drugs such as Cardiac and Diabetic care.

Microbiologist's as well as previous studies have shown that new organisms such as uncultured bacteria are likely to harbor new bacteria.99\% of the bacteria can't be grown under the current lab techniques. The resistance mechanism of known soil bacteria and the percentage of this soil bacteria that can be harvested is something to worry about and is more likely to cause a impeding crisis in health industry. Drs.Kim Lewis and Slava Epstein, of Northeastern University developed a new technique called iChip.iChip is a new and promising technology that can used to harvest previously unknown bacteria. Exploiting this new technology and therefore bacteria is likely to revive the natural drug discovery.

Teixobactin is a promising therapeutic candidate developed through iChip and it's effectiveness have been demonstrated against drug resistant pathogens in a number of animal model of infection. In experiments in mice, the researchers showed teixobactin was effective in treating animals infected with bacteria such as Mycobacterium tuberculous (which causes tuberculosis) and Staphylococcus aureus (which can infect people's skin and other tissues). Some strains of these bacteria are already resistant to one or more of antibiotics. Though still in very early stages of development, the discovery of a new class of antibiotics is good news.

Teixobactin kills bacteria by binding to lipids on their cell wall, causing their lysis. Most other antibiotics target proteins in bacteria, and the bacteria become resistant when the genes that code for those proteins mutate. But by targeting lipids instead of proteins it may take longer for the bacteria to develop resistance. Vancomycin works with similar mechanism of action and it took 30 years for developing resistance. Teixobactin is unable to penetrate Eleftheria terrae the organism that produces the antibiotic and thus may take even longer for resistance to develop. Clinical trials will be necessary to prove its effectiveness in humans.

\section{CONCLUSION}

Introduction of only two class of antibiotics since 1962 is a grim reminder that the current laboratory techniques cannot keep up with the pace infection and resistance are developed. 
iChip introduced by Dr.Slava Epstein and Dr.Kim Lewis and now licensed to NovoBiotic Pharmaceuticals is a novel way to harvest bacteria which previously was unculturable. Eleftheria terrae are one such bacteria which produced teixobactin effective against Gram positive superbugs commonly encountered. Unfortunately teixobactin is not effective against Gram negative pathogens.

However teixobactin will be a new drug to treat infection in people, provided it clears the rigorous clinical trials to mark it safe for humans. Though NovoBiotic pharmaceuticals had stated that the drug will be ready for clinical trial by 2017, it will be a long road before it is marketed. Whether Texiobactin is marketed or not, it will be the first of a new series of antibioticcs in its class.

\section{References}

1. Laura J. V. Piddock,. Teixobactin, the first of a new class of antibiotics discovered by iChip technology? $J$ Antimicrob Chemother, doi:10.1093/jac/dkv175

2. Ling LL, Schneider T, Peoples AJ et al. A new antibiotic kills pathogens without detectable resistance. Nature 2015; 517: 455-9.

3. Dharmesh Harvani,. The great plate count anomaly and the unculturable bacteria. www.worldwide journals. com, ijsr, file.

4. Cesar A. Arias, M.D., Ph.D., and Barbara E. Murray, M.D. A New Antibiotic and the Evolution of Resistance., N. Eng J Med 2015;1168-1170/March 19,2015;DOI:10,1056/NEJMcibr1500292.
5. Brooke Borel, www.popsci.com/ichip-new-way-findantibiotics-and-other-key-drugs.

6. Kaeberlain et al, "Isolating 'uncultivable' Microorganisms in Pure Culture in Simulated Natural Environment,"Science 296:5570 1127-1129(2002)

7. Nichols et al,'Use of iChip for High-Thoroughput**In Situ Cultivation of 'Uncultivable ' Microbial Specissm," Applied and Environmental Microbiology 76:8 2445-2450(2010)

8. Fleming, A. On the antibacterial action of cultures of a penicillium, with special reference to their use in the isolation of B. influenzae. Br. J. Exp. Pathol. 10, 226236 (1929)

9. Sakoulas, G. et al. Relationship of MIC and bactericidal activity to efficacy of vancomycin for treatment of methicillin-resistant Staphylococcus aureus bacteremia. J. Clin. Microbiol. 42, 2398-2402 (2004) PubMedArticle

10. Kollef, M. H. Limitations of vancomycin in the management of resistant staphylococcal infections. Clin. Infect. Dis. 45 (Suppl 3). S191-S195 (2007) PubMedArticle

11. Judy Stone, Texiobactin and iChip promise hope against antibiotic resistance, Forbes/Pharmacy \& Healthcare

\section{How to cite this article:}

Sandra Sagar et al (2017) ' The future of antibiotics?- ichip and teixobactin-a review', International Journal of Current Advanced Research, 06(04), pp. 3263-3266.

DOI: http://dx.doi.org/10.24327/ijcar.2017.3266.0241 